\title{
Dominio semántico y comprensión lectora en estudiantes de la Facultad de Educación de la Universidad Nacional Federico Villarreal, Lima - Perú
}

\author{
Semantic domain and reading comprehension in students of the Faculty of \\ Education at the \\ Universidad Nacional Federico Villarreal, Lima - Peru
}

Karina Cecilia Núñez Montalvan ${ }^{1}$

\section{RESUMEN}

El presente trabajo tiene por finalidad demostrar la relación entre el dominio semántico y la comprensión lectora en estudiantes de la Facultad de Educación de la Universidad Nacional Federico Villarreal de Lima, investigación efectuada en una muestra conformada por 70 sujetos a quienes se les suministró dos instrumentos: un cuestionario que mide el dominio semántico (Núñez, 2012) en los campos: vocabulario en contexto (sustantivos, adjetivos y verbos); sinónimos en contexto (sustantivos, adjetivos y verbos); antónimos (sustantivos, adjetivos y verbos); parónimos (sustantivos, adjetivos y verbos); homónimos (sustantivos, adjetivos y verbos); hiperónimos e hipónimos (sustantivos y verbos); marcadores textuales (conjunciones y palabras de enlace). Presenta validez y confiabilidad de 0,891 y una prueba de comprensión lectora (Tapia, 2012) que consta de 10 fragmentos de lecturas, en tres dimensiones: Literal (Información de hechos, definición de significados); Inferencial (Identificación de la idea central, interpretación de hechos, inferencia sobre el autor, inferencia sobre el contenido del fragmento, rotular o dar un titular al texto); Criterial (Valoración de los hechos presentados en los textos), con una fiabilidad de 0,66. Los resultados reportan la existencia de una relación significativa de 0,693 entre el dominio semántico y la comprensión lectora en los estudiantes. Respecto a las dimensiones, se observa una relación media $(0,453)$ entre el dominio semántico y la comprensión lectora en el aspecto Literal; una relación débil $(0,276)$ entre el dominio semántico y la comprensión lectora en el campo Inferencial; y, una relación baja $(0,369)$ entre el dominio semántico y la comprensión criterial.

\section{Palabras clave}

Dominio semántico, comprensión lectora, aspectos: Literal, Inferencial, Criterial.

\section{ABSTRACT}

The purpose of this paper is to demonstrate the relation between the semantic command and reading comprehension in students from the Education Faculty of Universidad Nacional Federico Villarreal from Lima. The research done on a sample of 70 people to whom two instruments were provided: a questionnaire that measures the semantic command (Nuñez, 2012) in the following fields: vocabulary in context (nouns, adjectives and verbs); synonyms in context (nouns, adjectives and verbs); antonyms (nouns, adjectives and verbs); paronyms (nouns, adjectives and verbs); homonyms (nouns, adjectives and verbs); hyperonyms and hyponyms (nouns and verbs); linkers (conjunctions and connectors). It is valid and reliable in 0,891 and there is also a reading comprehension test (Tapia, 2012). It consists of 10 pieces of reading, divided in three dimensions: Literal (Information about facts, definition of meanings); Inferential (Identification of

\footnotetext{
Bachiller en Educación. Docente de Educación Secundaria, especialidad de Comunicación, en la Institución Educativa de Mujeres Rímac y en Ed. Superior. Candidata para la obtención del grado de Magíster por la Universidad Nacional Mayor de San Marcos, mención: Docencia en el Nivel Superior.
} 
the main idea, understanding of facts, inference about the author, inference about the content of the pieces of reading, mark or give a title to the text); Criterial (assessment of the facts presented in the texts), it has reliability of 0,66. Results report the existence of a meaningful relation of 0,693 between the semantic domain and reading comprehension among students. Regarding dimensions, it is observed an average relation $(0,453)$ between the semantic domain and reading comprehension in the Literal level; a weak relation $(0,276)$ between the semantic domain and reading comprehension in the Inferential level; and, a low relation $(0,369)$ between the semantic domain and criterial comprehension.

\section{Keywords}

Semantic domain, reading comprehension: Literal level, Inferential level, Criterial level.

\section{INTRODUCCIÓN}

La investigación aborda la problemática recurrente del déficit semántico y limitaciones en la comprensión de textos académicos en universitarios. Se reporta que muchos estudiantes presentan limitaciones en comprender textos académicos en los niveles Literal, Inferencial y Criterial. Asimismo, tienen deficiencias en el dominio semántico, referido a vocabularios en contexto, sinónimos en contexto, antónimos, parónimos, homónimos, hiperónimos e hipónimos y marcadores textuales.

Muchas veces observamos cómo las palabras pueden caer en imprecisiones, si no les damos el sentido adecuado. Ello depende de dos contextos: el lingüístico, que lo constituyen las palabras que lo rodean, por ejemplo en "Alcánzame esa regla para subrayar". El contexto extralingüístico es la situación en la que se pronuncia una palabra; por ejemplo, dar el grito “iEs una orden!”, por ejemplo en un ámbito militar, no tiene la misma significancia que si vemos pasar un conjunto de frailes. Tampoco se debe confundir el significado de un término con su referente, que es la realidad concreta a la que designa en cada momento. La palabra transporte, que tiene un solo significado, puede tener varios referentes distintos (todos los medios para trasladarse). Dos expresiones con un significado totalmente distinto, como $\mathrm{mi}$ marido y el hijo de Betty pueden referirse a la misma persona.

En consecuencia, la semántica estudia el significado de las palabras, pero teniendo en cuenta el contexto en el que se presenta. Por ello, será fundamental que el lector entienda las referencias que se presentan en la oración para una buena comprensión del texto.
Con frecuencia, en las aulas universitarias, algunos docentes refieren comentarios como: "los estudiantes vienen, de la secundaria, con deficiente preparación", "no saben leer ni escribir", "tienen deficiencias para comunicarse de manera óptima". Este pensamiento de conformismo y buscar culpables hace que el problema se acentúe más en los estudiantes en cuanto al dominio semántico y la comprensión de textos académicos. La situación real es que muchos estudiantes han desarrollado limitadamente capacidades fundamentales que les sirvan para continuar estudios superiores con la optimización requerida en cuanto al dominio semántico y la comprensión lectora.

En este contexto, el desarrollo de habilidades para la lectura comprensiva de textos especializados constituye un punto gravitante en el desarrollo de los sistemas de enseñanza en la educación terciaria, toda vez que el lenguaje escrito es medio preferente para la creación, fijación y transmisión del conocimiento disciplinar (Parodi, 2008). E experto y el neófito no leerán de igual forma un mismo texto. La importancia de la lectura con propósitos académicos radica en que este proceso forma parte de la práctica social de transmisión de conocimiento dentro de una entidad altamente institucionalizada y convencionalizada, que crea restricciones y requisitos, y que demanda de los lectores la apropiación de las convenciones que regulan la comunidad. (Carlino, 2005).

Varios son los autores que han señalado distintos procesos de comprensión que intervienen en la lectura. Aquí, se señalan los de Alliende y Condemarín (2002, citado en Menacho, 2010) que, a su vez, se basan en la taxonomía de Barret y que se han utilizado en las distintas evaluaciones sobre comprensión lectora. 


\section{Dominio semántico}

Bunge (2002) define la semántica como "la teoría del significado, la verdad y conceptos afines" (p. 21). Sostiene que la semántica, en cuanto es determinante en cualquier actividad, relación o situación cognitiva para comprender el significado de cualquier elemento, tiene dos visiones: aquella que es ofrecida o propuesta inicialmente y la que es percibida por el sujeto al momento de interactuar. Por su parte, Muñoz (2006) refiere que la semántica es la dimensión del lenguaje que relaciona los elementos lingüísticos, nombres, predicados, oraciones, con los objetos, las acciones y propiedades y los hechos del mundo. La semántica tiene que ver con nuestra capacidad de representarnos el mundo mediante símbolos.

Cuando un sujeto interactúa con sus semejantes, tiene muchos motivos para tratar de controlar la impresión que ellos reciban de la situación. El contenido específico de una actividad presentada por un individuo y el rol que este desempeña en esas actividades en un contexto social, están determinados por dos actividades significantes: en el primer caso, se tiene lo que corresponde a la intención planificada por el individuo para llevar a cabo y, en el otro, por lo que infieren las otras personas que participan o a las que está dirigida la actividad.

Esto sucede en cualquier contexto, más aún en uno educativo, donde la información ofrecida a los estudiantes por su docente está estructurada bajo el enfoque semántico de este último, pero, sin duda alguna, la semántica con la que esa misma información es percibida por cada uno de los estudiantes puede ser significativamente diferente. La semántica, en este contexto, determina la correcta aprehensión del significado de la actividad y sus contenidos, llegando a promover la negociación de los significados particulares entre el docente y sus estudiantes para lograr un punto de vista semánticamente común a todos los actores del proceso.

Al respecto, es preciso recordar lo que es un símbolo y cuáles son sus componentes. Un símbolo o signo tiene una estructura triangular: a) el significante: es un objeto lingüístico arbitrario, una secuencia de grafías o fonemas que tiene una existencia física; por ejemplo: la palabra 'mesa'; b) el significado: es la intensión del significante y es un concepto o categoría que expresa las propiedades generales y significativas de un objeto de la realidad que denotamos con el significante. Por ejemplo, la palabra 'mesa' significa un tablero con cuatro patas capaces de soportar un peso o una fuerza. El significado nos permite localizar la referencia, el objeto del que habla el significante; c) la referencia o extensión es el objeto real del mundo representado por el significante. Las referencias de los posibles tipos de significantes del lenguaje serían las siguientes: El nombre refiere a un objeto del mundo; el predicado refiere a una propiedad del mundo; $y$, la oración refiere a un valor de verdad (Muñoz, 2006).

\section{Comprensión lectora}

Campanario y Otero (2000) definen la comprensión lectora como el proceso para elaborar los significados de las ideas relevantes del texto y relacionarlas con las que ya se tienen; proceso en el cual el lector interactúa con el texto que lee. Por su lado, García (2003) sostiene que "para comprender un texto escrito se realizan variadas operaciones intelectuales de gran complejidad, y enfatiza que "no basta con la decodificación de signos gráficos o letras escritas, el reconocimiento de palabras y lo que esto significa (procesos léxicos). Estos son procesos necesarios pero no suficientes para la lectura comprensiva". (p. 87)

Además de los procesos de reconocimiento, se requieren procesos de orden sintáctico y semántico; los primeros están referidos a poner en relación las palabras para constituir unidades mayores, como las frases y las oraciones; los segundos hacen referencia a procesos para captar el significado de las oraciones y del contenido del texto, integrándolo con los conocimientos previos del lector.

En esa línea de ideas, Sánchez (2001) argumenta que el proceso de lectura implica dos procesos cognoscitivos complejos: primero se encuentran los procesos relacionados con el reconocimiento de las palabras, que exigen la habilidad para pasar de la ortografía de las palabras a su fonología y significado; luego, se describen los procesos relacionados con la comprensión del lenguaje escrito, para lo cual es necesario reconocer el carácter comunicativo de la lectura. 
Es preciso también considerar las fases para desarrollar la comprensión lectora. Mendoza y Briz (2003) postulan: antes, durante y después de la lectura. "Antes de la lectura, se produce la activación de los conocimientos previos, así como la rememoración de vivencias y experiencias con relación al texto que desempeñarán un papel importante en la comprensión, y durante la lectura, el alumnado participa activamente en la construcción del texto, verificando si se cumplen las previsiones realizadas por él”. (p. 114)

\section{Nivel literal}

En este nivel, el lector es capaz de reconocer todo lo que está explícito en el texto; por lo que podrá localizar información relevante y distinguirla de la complementaria. También será capaz de extraer datos como los nombres de los personajes, hechos importantes, tiempoy espacio; identificar algunas relaciones sencillas de causa-efecto, encontrar el sentido de las palabras en cuanto a su significado. Serían claros indicios de que el lector domina este nivel, si puede recordar lo leído y expresarlo, así también si utiliza el vocabulario que ha captado en el texto.

\section{Nivel inferencial}

Se espera que en este nivel el lector sea capaz de interactuar con el texto; pues puede inferir la postura del autor, relaciones lógicas, lenguaje figurativo, valores o ideología implícita en el texto. Todo ello, debe entenderse, se da conjugando su experiencia previa con la comprensión del texto.

Serían indicios de una comprensión inferencial que el lector pueda elaborar interpretaciones sobre los valores, el propósito del texto, características de personajes a partir de la lectura y teniendo en cuenta sus conocimientos.

\section{Nivel criterial}

En este nivel, el lector será capaz de emitir juicios y valoraciones luego de la lectura, estos pueden tener una carga subjetiva, pero que parte del análisis y la comprensión del texto. Serían indicios de una comprensión en el nivel criterial, si el lector puede reflexionar sobre la información del texto, evaluándola y contrastándola con sus conocimientos o por medio de otras fuentes, ello en el plano tanto del fondo como de la forma.

\section{MATERIAL Y MÉTODOS}

\section{Dominio semántico}

Los resultados de la investigación reportan que el $41,4 \%$ de los universitarios se ubica en el nivel medio, seguido del 34,3\% que se halla en el nivel alto, y un $24,3 \%$ que está en el nivel bajo, lo que indica que las dos terceras partes de los estudiantes no presenta, en términos generales, un dominio semántico óptimo en contextos comunicacionales.

\section{Comprensión lectora}

El $70 \%$ de los estudiantes se ubica en el nivel medio en comprensión lectora, seguido del $24,3 \%$ que comprende en un nivel óptimo y solo el $5,7 \%$ en un nivel bajo. Esto indica que la mayoría de los estudiantes comprende medianamente textos académicos de uso frecuente en las aulas universitarias.

\section{Correlación entre dominio semántico y comprensión lectora}

Existe una relación estadísticamente significativa $(\mathrm{r}=0,693$, donde $\mathrm{p}<\mathrm{de} 0,01)$ entre el dominio semántico y la comprensión lectora en los estudiantes. Al tenerse una significancia de 0,000 la misma que se encuentra dentro del valor permitido $(0,01)$ se acepta la hipótesis general en el sentido siguiente: Existe relación positiva y significativa entre el dominio de la semántica y la comprensión lectora en los estudiantes de la Facultad de Educación de la Universidad Nacional Federico Villarreal.

Se reporta una relación estadísticamente significativa $(r=0,693$, donde $\mathrm{p}<\mathrm{de} 0,01)$ entre el dominio semántico y la comprensión lectora en los estudiantes. Al tenerse una significancia de 0,000 , la misma que se encuentra dentro del valor permitido $(0,01)$ se acepta la hipótesis general en el sentido siguiente: Existe relación positiva y significativa entre el dominio de la semántica y la comprensión lectora en los estudiantes.

Se evidencia una relación débil $(r=0,276$, donde $\mathrm{p}<\mathrm{de} 0,05)$ entre el dominio semántico y la comprensión lectora en el nivel inferencial en los estudiantes. Al tenerse una significancia de 0,000 , la misma que se encuentra dentro del valor permitido $(0,05)$, se acepta la segunda hipótesis específica en el sentido siguiente: El dominio 
semántico se relaciona positiva, aunque débilmente, con la comprensión lectora en el nivel inferencial en los estudiantes.

Existe una relación débil $(\mathrm{r}=0,369$, donde $\mathrm{p}<\mathrm{de} 0,01$ ) entre el dominio semántico y la comprensión lectora en el nivel criterial en los estudiantes. Al tenerse una significancia de 0,000, la misma que se encuentra dentro del valor permitido $(0,01)$, se acepta la tercera hipótesis específica en el sentido siguiente: Existe una relación positiva aunque débil entre el dominio semántico y la comprensión lectora en el nivel criterial en estudiantes.

\section{DISCUSIÓN DE RESULTADOS}

Los resultados de la investigación reportan la existencia de una relación positiva y significativa entre el dominio semántico y la comprensión lectora $(0,693)$ en estudiantes de la Facultad de Educación de la Universidad Nacional Federico Villarreal. Esto indica que los estudiantes que en su mayoría comprenden medianamente están en relación directa con el dominio que tienen. No obstante, se determina que los estudiantes presentan bajo nivel de comprensión lectora.

Estos datos coinciden con lo hallado por Echavarría \& Gastón (2000) quienes demuestran que los estudiantes presentan dificultades en la comprensión de los textos a nivel de la selección y jerarquización de la información relevante (macroestructura) y en la captación de la intencionalidad comunicativa del autor, que se refleja en la estructura del texto (superestructura). La dificultad en la comprensión se da, bien porque se carece de los conocimientos necesarios o porque no se activan los conocimientos relevantes.

En lo que respecta a la primera dimensión, los resultados reportan una relación media $(r=453)$ entre el dominio semántico y la comprensión lectora en el nivel inferencial. Asimismo, se reporta una relación débil $(0,276)$ entre el dominio semántico y la comprensión lectora en el nivel inferencial; $y$, una relación moderada $(0,369)$ entre el dominio semántico y la comprensión lectora en el nivel criterial.
Al respecto, Ochoa y Aragón (2004) encontraron una relación significativa y positiva entre el funcionamiento metacognitivo de estudiantes universitarios y los niveles de comprensión de artículos científicos tipo ensayo teórico y reporte de investigación, de tal modo que a mayor nivel metacognitivo, mayor nivel de comprensión lectora, y viceversa. Los sujetos fueron clasificados de acuerdo con sus desempeños metacognitivos en los procesos de planificación monitoreo en seis niveles: lector no regulado, ligeramente regulado, parcialmente regulado, medianamente regulado, autorregulado y muy regulado.

En esa misma línea, Martínez (2008) reporta la existencia de una tendencia de bajo nivel de conocimientos y uso de estrategias lectoras y un nivel inferior en comprensión de lectura de los estudiantes que orienta a establecer una posible relación entre el nivel de conocimientos y uso de estrategias lectoras con el nivel de comprensión de lectura. Asimismo, hay una relación directa entre el nivel de conocimiento y uso de estrategias lectoras; existe diferencia significativa entre los promedios totales de los estudiantes provenientes de instituciones educativas privadas y públicas. Sin embargo, otros aspectos como la experiencia profesional y la institución de formación docente no se encuentran vinculados con dicha relación. Se demuestra que hay correlación entre el nivel de conocimiento de estrategias lectoras y el nivel de comprensión lectora. Mac Dowal (2009) reporta la existencia de una relación significativa, las estrategias de aprendizaje y la comprensión lectora en los estudiantes. Es decir, los estudiantes que aplican diversas estrategias de aprendizaje obtienen mejores resultados en la comprensión de textos académicos. Esta relación se da con las estrategias de adquisición de información, codificación de información, recuperación de información y apoyo al procesamiento de la información.

\section{CONCLUSIONES}

Los resultados de la investigación reportan la existencia de una relación positiva y significativa entre el dominio semántico y la comprensión lectora en los estudiantes de la Facultad de Educación de la Universidad Nacional Federico Villarreal, Lima. Esto significa la comprensión lectora que presentan los alumnos 
mayoritariamente en un nivel medio. También se reporta la existencia de una relación media entre el dominio semántico y la comprensión lectora en la dimensión nivel literal. Se encontró una relación débil entre el dominio semántico y la comprensión lectora en la dimensión nivel inferencial. Asimismo, hay una relación baja entre el dominio semántico y la comprensión lectora en la dimensión nivel criterial, en estudiantes de la Facultad de Educación de la Universidad Nacional Federico Villarreal. 


\section{REFERENCIAS}

Bunge, M. (2002) Ser, saber y hacer. México: Paidos.

Campanario, J. M. y Otero, J. (2000) Más allá de las ideas previas como dificultades de aprendizaje: las pautas de pensamiento, las concepciones epistemológicas y las estrategias metacognitivas de alumnos de ciencias. Revista Enseñanza de las Ciencias, 18 2: 161-169.

Carlino, P. (2005) Escribir, leer y aprender en la Universidad. Buenos Aires: Fondo de Cultura Económica.

Echavarría, M. \& Gastón, I. (2000) Dificultades de comprensión lectora en estudiantes universitarios. Implicaciones en el diseño de programas de intervención. Revista de Psicodidáctica, Universidad del País Vasco, 010: 1-16.

García, E. (2003) La comprensión de textos, modelo de procesamiento y estrategias de mejora. Revista Didáctica, 5: 87-117.

Mac Dowall, E. (2009) Relación entre las estrategias de aprendizaje y la comprensión lectora en alumnos ingresantes de la Facultad de Educación de la Universidad Nacional Mayor de San Marcos. (Tesis de maestría). Universidad Nacional Mayor de San Marcos, Lima.

Martínez, T. (2008) Comprensión de lectura y estrategias lectoras en docentes egresados de Institutos Pedagógicos. (Tesis de maestría). Universidad Nacional Mayor de San Marcos, Lima.

Menacho, J.C. (2010) Metodología de aprendizaje cooperativo como propuesta de innovación en la enseñanza de semiología general e interpretación de exámenes auxiliares. (Tesis de maestría). Universidad Nacional Mayor de San Marcos, Lima.

Mendoza, A. y Briz, E. (2003) Didáctica de la Lengua y la Literatura. España: Editorial Prentice Hall.

Muñoz, C. (2006) Semántica cognitiva: Modelos cognitivos y espacios mentales. Revista de Filosofía a Parte Rei, n 43, pp. 1-28.

Ochoa, S. \& Aragón, L. (2004) Funcionamiento metacognitivo de estudiantes universitarios durante la lectura de artículos científicos. Recuperado el 28 de abril de 2013 de: http://redaly c.uaemex.mx/pdf/647/64760303.pdf.

Parodi, G. (2008) Géneros Académicos y Géneros Profesionales: Accesos Discursivos para Saber y Hacer. Valparaíso: Ediciones Universitarias de Valparaíso.

Sánchez, E. (2001) El lenguaje escrito y sus dificultades: una visión integradora. En: Marchesi, A., Coll, C. \& Palacios, J. (Comps.). Desarrollo psicológico y educación. Tomo III. Trastornos del desarrollo y necesidades educativas especiales. Madrid: Alianza Editorial.

Fecha de recepción: 30-05-17

Fecha de aceptación: 11-09-17 